\title{
Intercellular adhesion molecule-1 expression and serum levels as markers of pre-clinical atherosclerosis in polycystic ovary syndrome
}

Nearmeen M. Rashad ${ }^{1 *}$ (D, Amal S. El-Shal ${ }^{2}$, Hala G. Abomandour ${ }^{3}$, Amr Mostafa Kamel Aboelfath ${ }^{4}$, Mohamed el sayed Rafeek ${ }^{4}$, Mohammad Samir Badr ${ }^{4}$, Ayman E. Ali', Mohammed S. Yousef',

Maha Abdelhamid Fathy ${ }^{5}$ and Mustafa taha Abdelfattah Sharaf el din ${ }^{4}$

\begin{abstract}
Background: Polycystic ovary syndrome (PCOS) is a common reproductive endocrine disorder characterized by obesity, hyperandrogenism, and insulin resistance. Intercellular adhesion molecule-1 (ICAM-1) is a proinflammatory and proatherogenic cytokine which is associated with atherosclerosis, insulin resistance, and cardiovascular disease (CVD). The pathogenesis of PCOS is not precisely known. Thus, the purpose of this study was to investigate the potential role of ICAM-1 expression and serum ICAM-1 concentrations in pathogenesis of PCOS. Moreover, we aimed to evaluate the possible relationship between ICAM-1 gene expression with carotid intima-media thickness as well as clinic-morphological features of PCOS.
\end{abstract}

Methods: This case control study enrolled 180 patients with PCOS and 120 controls groups and they were stratified according to their fasting plasma glucose (FPG) into three subgroups; normal glucose tolerance (NGT) $[n=75]$, those with impaired glucose tolerance (IGT) $[n=65]$, and 40 patients with type 2 diabetes mellitus (T2DM). Circulating ICAM-1 expression levels were determined by real time polymerase chain reaction (RT-PCR). Serum ICAM-1 concentrations were measured using enzyme-linked immunosorbent assay (ELISA).

Results: Our results revealed that PCOS patients had higher values of ICAM-1expression and serum levels. Among PCOS patients, T2DM patients had the highest values of ICAM-1 expression and serum levels compared to IGT and NGT subgroups. The ICAM-1 expression and serum levels were significantly positive correlated with cardiovascular risk and PCOS phenotypes. Linear regression test showed that HOMA-IR was the main predictors of serum ICAM-1 levels in PCOS. Receiver operating characteristic curve (ROC) analysis revealed that, the power of ICAM-1 expression levels was higher than serum ICAM-1 in diagnosis of PCOS and in differentiating T2DM from IGT and NGT subgroups. Interestingly, combination of both ICAM-1 expression and serum levels improved the diagnostic role of serum ICAM-1.

Conclusion: ICAM-1 expression and serum levels were higher in women with PCOS compared to control group also, there was a strong independent association between higher ICAM-1 expression and serum levels with cardiovascular risks in PCOS group.

Keywords: Cardiovascular, Polycystic ovary syndrome, Intercellular adhesion molecule-1, Type 2 diabetes mellitus, Enzyme-linked immunosorbent assay

\footnotetext{
* Correspondence: nrashad78@yahoo.com; n.rashad@zu.edu.eg

'Internal Medicine Department, Faculty of Medicine, Zagazig University,

Zagazig, Egypt

Full list of author information is available at the end of the article
}

(c) The Author(s). 2019 Open Access This article is distributed under the terms of the Creative Commons Attribution 4.0 International License (http://creativecommons.org/licenses/by/4.0/), which permits unrestricted use, distribution, and reproduction in any medium, provided you give appropriate credit to the original author(s) and the source, provide a link to the Creative Commons license, and indicate if changes were made. The Creative Commons Public Domain Dedication waiver (http://creativecommons.org/publicdomain/zero/1.0/) applies to the data made available in this article, unless otherwise stated. 


\section{Introduction}

Polycystic ovary syndrome (PCOS) is the commonest endocrinopathy of premenopausal women characterized by both reproductive and metabolic abnormalities. It affects $5-10 \%$ of reproductive-age women [1] Women with PCOS are characterized by chronic anovulation, hyperandrogenism [2], insulin resistance (IR), dyslipidemia, obesity, type 2 diabetes mellitus (T2DM) [3], low-grade chronic inflammation, and increased oxidative stress [4].

The exact pathophysiologic mechanism of PCOS remains still unclear. There is considerable evidence for the involvement of low-grade chronic inflammation and endothelial dysfunction in the pathogenesis of PCOS and its associated features such as insulin resistance (IR), dyslipidemia, and atherosclerosis [5].

Atherosclerosis is an important pathologic cause of cardiovascular diseases (CVD). Additionally, CVD and cerebrovascular diseases are the leading causes of mortality in humans and can have significant impacts on morbidity. Atherosclerosis starts by preclinical increase in the thickness of the internal and medial membrane of the arterial wall which has been related to higher coronary heart disease and stroke rates [6]. Carotid intimamedia thickness (CIMT) measured by ultrasound is a noninvasive, safe, low-cost, reproducible, and well validated marker of preclinical atherosclerosis $[7,8]$.

Intercellular adhesion molecule-1 (ICAM-1), (CD54), a 90- $\mathrm{kDa}$ cell surface glycoprotein, is involved in the firm adhesion of leukocytes to endothelium and their transendothelial migration to sites of inflammation [9]. An important event in the initiation of atherosclerosis is the adhesion of circulating monocytes to the activated endothelial cells and their subsequent transendothelial migration into the subendothelial space. These processes depend on the coordinated expression and activation of several cellular adhesion molecules (CAMs) such as vascular cell adhesion molecule1 (VCAM-1), ICAM-1, Eselectin and their counter-receptors on monocytes [10]. Meanwhile, the expression of endothelial cell adhesion proteins is induced by proinflammatory cytokines such as tumor necrosis factor- $\alpha$ (TNF- $\alpha)$ and interleukin-1 (IL-1 $\beta)$ and by specific oxidants $[10,11]$.

The concept of the critical role of adhesion molecules in endothelial dysfunction has been reported [12]. It has been shown recently that cellular adhesion molecule levels, including ICAM-1 suggested to be increased in patients with PCOS [13].

PCOS is a highly complex endocrine disorder and phenotypically is heterogeneous. The purpose of this study was to investigate the potential role of ICAM-1 serum and expression levels. Moreover, we aimed to explore role of ICAM-1 in early detection of cardiovascular risk in patients with PCOS, and to evaluate the possible relationship between ICAM-1 gene expression with carotid intima- media thickness as well as clinic-morphological features of PCOS.

\section{Subject and method}

This case control study included 300 unrelated subjects. One hundred eighty PCOS patients recruited from Outpatient Clinics of the Endocrinology Unit of Internal Medicine and Obstetrics and Gynecology Departments, Faculty of Medicine, Zagazig University, Egypt and 120 healthy women matched to PCOS cases as regard age, and body mass index (BMI). PCOS patients were then stratified into three subgroups according to their fasting plasma glucose (FPG) based on the American Diabetes Association criteria reported in 2016, [14] those with normal glucose tolerance (NGT; 75 women), those with impaired glucose tolerance (IGT; 65 patients), and 40 patients with type 2 diabetes mellitus (T2DM). The diagnosis of PCOS was based on the 2004 revised Rotterdam criteria [15]. All patients were subjected to thorough history taking, full clinical assessment, and anthropometric measures of obesity. Ovarian volume and antral follicular count (AFC) were evaluated by transvaginal ultrasound (TVS). Body compositions including fat mass (FM) and fat free mass (FFM) were measured by Dual-energy X-ray absorptiometry (DEXA).

Exclusion criteria for all women included a history of hyperandrogenic states (such as non-classical congenital adrenal hyperplasia, androgen secreting tumors, Cushing's syndrome, 21-hydroxylase deficiency, or hyperprolactinemia), diabetes mellitus (DM), hypertension, liver, kidney, or thyroid diseases.

\section{Ethics approval and consent to participate}

A written informed consent was taken from all of the participants after explaining details and benefits as well as risks to them. The ethical committee of Faculty of Medicine, Zagazig University approved this study.

\section{Sampling of blood}

The blood samples of all study's subjects were drawn after an overnight fast and divided into 3 portions: $1 \mathrm{ml}$ of whole blood was collected into EDTA tubes, for RNA extraction and $\mathrm{HbA} 1 \mathrm{c} ; 1 \mathrm{ml}$ of blood was collected into potassium oxalate and sodium fluoride containing tubes for fasting blood glucose (FBG) and 2-h plasma glucose. Sera were separated from remaining sample part and stored at $-20^{\circ} \mathrm{C}$ until analysis.

\section{Biochemical analysis}

We determined FBG and 2-h plasma glucose levels using the glucose oxidase method (Spinreact, Girona, Spain). Total cholesterol, HDL cholesterol, and triglycerides levels were measured by routine enzymatic methods (Spinreact, 
Girona, Spain). The LDL cholesterol level was calculated using the Friedewald formula [16].

\section{Immunochemical assays}

Fasting serum insulin (FSI) levels were determined by high-sensitivity linked immunosorbent assay (ELISA) kit provided by (Biosource Europe S.A., Nivelles, Belgium). Homeostasis model assessments of insulin resistance (HOMA-IR) and B-cell function (HOMA-B) were calculated. Serum ICAM-1 concentrations were measured with a sandwich ELISA using the human $\mathrm{RayBio}^{\circ} \mathrm{Hu}$ man sICAM-1 ELISA kit (RayBio, Nacross, USA).

\section{RNA extraction, CDNA synthesis and real time PCR for ICAM-1 mRNA gene expression}

Total RNA was extracted from whole blood using QIAamp ${ }^{\circ}$ RNA Blood MiniKit following the manufacturer's protocol (QIAGEN, Valencia,USA). The quantity and purity of RNA was confirmed by optical density (OD) at 260 and $280 \mathrm{~nm}$ using an ultraviolet spectrometer, with acceptable RNA purity ranging from 1.8 to 2.1 . The ratio of absorbance values at 260 and $280 \mathrm{~nm}$ indicated an estimate of RNA purity. The extracted RNA was reverse transcribed by (QuantiTect Reverse Transcription Kit, QIAGEN, Valencia, CA, USA) as recommended by the manufacturer. Expression levels of ICAM-1 mRNA were determined by quantitative real time PCR (RT-PCR) according to previous study [17]. The ICAM-1 primers sequence were: forward primer; 5'AGGCCACCCCAGAGGACA AC $-3^{\prime}$ and reverse primer $3^{\prime}$ - CCCATTATGACTGCGGCTGCTA $-5^{\prime}$, the product was 406 bp; a housekeeping gene $\beta$-actin forward primer: $5^{\prime}$ ATGTTTGAGACCTTCAACAC -3 ', and reverse primer: 5'-CACGTCACACTTCATGATGG-3', of 489 bp length; was used as an internal control. The expression of candidate gene mRNA was measured using a StepOne ${ }^{\text {tw }}$ System (Applied Biosystems). The PCR was performed in 25 final reaction volume using QuantiTect SYBR ${ }^{\circ}$ Green PCR Kits (QIAGEN, Valencia, CA, USA) containing $12.5 \mu \mathrm{l} 2 \mathrm{X}$ SYBR Green PCR Master Mix, $1 \mu \mathrm{M}$ of each primer and $2 \mu \mathrm{l}$ cDNA. The following conditions were used: denaturation at $95^{\circ} \mathrm{C}$ for $5 \mathrm{~min}$ (hot start), 40 cycles of denaturation $\left(95^{\circ} \mathrm{C}\right.$ for $10 \mathrm{~s}$ ), and combined annealing and extension annealing $\left(60^{\circ} \mathrm{C}\right.$ for $\left.30 \mathrm{~s}\right)$. The expression of ICAM- 1 was reported as the $\Delta \mathrm{Ct}$ value, which was calculated by subtracting the $\mathrm{CT}$ values of $\beta$-actin from the CT values of the target ICAM-1.

\section{Statistical analysis}

Data analyses were done with statistical package for the social sciences software (SPSS Version 21, Chicago, Illinois). Data were expressed as mean \pm standard deviation (SD). The relationships of ICAM-1 serum and expression level with clinical and laboratory parameters among PCOS patients were tested with the Pearson correlation. Receiver operating characteristic (ROC) analysis was performed to assess the diagnostic power of ICAM-1 serum and expression level. Linear regression analysis was done to detect the main predictors of serum ICAM1 in PCOS group. We considered $P$ to be significant at $<0.05$ with a $95 \%$ confidence interval $(\mathrm{CI})$.

\section{Results \\ Clinical and laboratory characteristics of the study subjects}

In PCOS group, patients had significantly higher values of systolic blood pressure (SBP), total cholesterol (TC), triglycerides (TG), low-density lipoprotein (LDL)-cholesterol, FPG, 2-h plasma glucose, FSI, hemoglobin A1c (HbA1c), homeostatic model assessment-IR (HOMAIR), high sensitivity C-reactive protein (hsCRP), white blood cell (WBC) count, and CIMT when compared to control group. Additionally, as expected patients with PCOS had significantly higher values of clinical and biochemical characteristic of PCOS; hirsutism score, ovarian volume, AFC, FSH, LH, LH/FSH, DHEA-S, androstenedione, total testosterone, and FAI compared control group. On the contrary, patients with PCOS had significantly lower levels of HDL, HOMA $-\beta$, and SHBG when compared with controls (Table 1).

\section{Clinical and laboratory characteristics of women with PCOS}

Among PCOS group, T2DM patients had significant higher values of body composition parameters; waist/hip ratio, FMI\% and FFMI\%. Also, systolic and diastolic blood pressure as well as, TG, FPG, 2-h plasma glucose, FSI, HbA1c, HOMA-IR, hs-CRP, WBC count, and CIMT values were increased when compared to NGT and IGT groups. In addition, PCOS phenotypes; ovarian volume, AFC, total testosterone, FAI, LH and DHEA-S were significantly higher in T2DM patients compared to NGT and IGT PCO subgroups. On the contrary, we detected significant lower SHBG and HOMA- $\beta$ levels in T2DM patients than in those NGT or IGT patients.

In IGT subgroup, we observed significant higher levels of systolic and diastolic blood pressure as well as waist/hip ratio, FMI\%, FFMI\%, FPG, 2-h plasma glucose, FSI, HbA1c, HOMA-IR, hs-CRP, and CIMT s than in those NGT group. Moreover, PCOS phenotypes values; ovarian volume, AFC, total testosterone, FAI, FSH, LH, LH/FSH and DHEA-S were significantly higher in IGT than NGT. On the other hand, we demonstrated significant lower values of SHBG, HDL, and HOMA-B in IGT compared to NGT (Table 2).

\section{Comparison of expression and serum levels of ICAM-1 in studied groups}

Regarding expression levels of ICAM-1, PCOS patients $(4.57 \pm 2.16)$ had significantly higher levels compared to control group $(0.75 \pm 0.48)$ (Fig. 1a). Among PCOS group, 
Table 1 Clinical, anthropometric and laboratory characteristics of studied groups

\begin{tabular}{|c|c|c|c|}
\hline & $\begin{array}{l}\text { Control group (mean } \pm \text { SD) } \\
(n=120)\end{array}$ & $\begin{array}{l}\text { PCO patients (mean } \pm \text { SD) } \\
(n=180)\end{array}$ & $P$ \\
\hline Age (years) & $31.38 \pm 7.88$ & $31.95 \pm 7.42$ & 0.527 \\
\hline Systolic blood pressure (mm Hg) & $127.6 \pm 5.62$ & $131.06 \pm 7.021$ & $<0.001^{*}$ \\
\hline Diastolic blood pressure (mm Hg) & $86.2 \pm 3.94$ & $87.31 \pm 6.074$ & 0.076 \\
\hline Hirsutism score & $5.53 \pm 0.472$ & $13.54 \pm 2.51$ & $<0.001^{*}$ \\
\hline Body mass index $(\mathrm{kg} / \mathrm{m} 2)$ & $30.28 \pm 57$ & 31.889 .99 & 0.103 \\
\hline Waist/hip ratio & $1.11 \pm 0.26$ & $1.14 \pm 0.30$ & 0.392 \\
\hline FMI $(\mathrm{kg} / \mathrm{m} 2)$ & $7.66 \pm 1.45$ & $7.86 \pm 1.579$ & 0.260 \\
\hline FFMI (kg/m2) & $21.81 \pm 4.13$ & $22.42 \pm 4.47$ & 0.233 \\
\hline Ovarian volume & $4.33 \pm 1.1$ & $10.64 \pm 1.3$ & $<0.001^{*}$ \\
\hline AFC & $5.33 \pm 1.1$ & $12.14 \pm 1.4$ & $<0.001^{*}$ \\
\hline Total cholesterol (mg/dL) & $179.47 \pm 19.7$ & $198.8 \pm 52.38$ & $<0.001^{*}$ \\
\hline Triglycerides (mg/dL) & $150.23 \pm 20.84$ & $187.6 \pm 62.14$ & $<0.001^{*}$ \\
\hline LDL cholesterol (mg/dL) & $117.48 \pm 19.05$ & $165.98 \pm 60.15$ & $<0.001^{*}$ \\
\hline HDL cholesterol (mg/dL) & $56.58 \pm 6.04$ & $39.2 \pm 13.63$ & $<0.001^{*}$ \\
\hline Fasting plasma glucose (mg/dL) & $87.65 \pm 9.14$ & $108.2 \pm 21.62$ & $<0.001^{*}$ \\
\hline 2-h plasma glucose (mg/dL) & $127.6 \pm 24.2$ & $163.67 \pm 41.7$ & $<0.001^{*}$ \\
\hline $\mathrm{HbA} 1 \mathrm{c}(\%)$ & $5.82 \pm 0.182$ & $6.35 \pm 0.977$ & $<0.001^{*}$ \\
\hline Fasting serum insulin (IU/mL) & $7.14 \pm 1.427$ & $20.05 \pm 7.3$ & $<0.001^{*}$ \\
\hline HOMA-IR & $1.55 \pm 0.39$ & $4.63 \pm 1.87$ & $<0.001^{*}$ \\
\hline HOMA- $\beta$ & $169.7 \pm 18.14$ & $122.01 \pm 43.6$ & $<0.001^{*}$ \\
\hline FSH (mIU/mL) & $6.17 \pm 1.53$ & $6.6 \pm 1.26$ & $<0.001^{*}$ \\
\hline $\mathrm{LH}(\mathrm{mlU} / \mathrm{mL})$ & $6.95 \pm 0.646$ & $10.3 \pm 1.23$ & $<0.001^{*}$ \\
\hline LH/FSH & $1.24 \pm 0.54$ & $1.6 \pm 0.278$ & $<0.001^{*}$ \\
\hline SHBG (nmol/L) & $53.58 \pm 6.04$ & $28.8 \pm 4.71$ & $<0.001^{*}$ \\
\hline DHEA-S (mg/mL) & $1.22 \pm 0.33$ & $2.37 \pm 0.316$ & $<0.001^{*}$ \\
\hline Androstenedione (ng/mL) & $1.41 \pm 0.508$ & $1.62 \pm 0.557$ & $<0.001^{*}$ \\
\hline Total testosterone $(\mathrm{ng} / \mathrm{mL})$ & $0.67 \pm 0.18$ & $1.1 \pm 0.218$ & $<0.001^{*}$ \\
\hline Free androgen index & $1.03 \pm 0.36$ & $2.56 \pm 1.22$ & $<0.001^{*}$ \\
\hline hs-CRP ( $\mu \mathrm{g} / \mathrm{ml})$ & $2.61 \pm 0.51$ & $5.56 \pm 0.98$ & $<0.001^{*}$ \\
\hline WBC count $\left(\right.$ cell $\left.\times 10^{3} / \mu \mathrm{l}\right)$ & $4.74 \pm 0.397$ & $6.97 \pm 2.31$ & $<0.001^{*}$ \\
\hline CIMT (mm) & $0.69 \pm 0.06$ & $1.18 \pm 0.46$ & $<0.001^{*}$ \\
\hline Serum ICAM-1 (ng/ml) & $87.8 \pm 49.5$ & $445.17 \pm 184.95$ & $<0.001^{*}$ \\
\hline Relative ICAM expression levels & $0.75 \pm 0.48$ & $4.57 \pm 2.16$ & $<0.001^{*}$ \\
\hline
\end{tabular}

FSI fasting serum insulin, FPG fasting plasma glucose, AFC antral follicle cells, FMI fat mass index, FFMI fat free mass index, HOMA-IR homeostasis model assessments of insulin resistance, $D H E A$ dehydroepiandrosterone; ${ }^{*} P<0.05$ when compared with control group

T2DM patients $(6.05 \pm 2.119)$ had significantly higher expression levels of ICAM-1 compared to IGT $(5.7 \pm 1.52)$ and NGT (2.68 \pm 0.85$)$ (Fig. 2a).

Regarding serum ICAM-1 levels, PCOS patients $(445.17 \pm 184.95 \mathrm{ng} / \mathrm{ml})$ had significantly higher levels compared to control group $(87.8 \pm 49.5 \mathrm{ng} / \mathrm{ml})$ (Fig. 2a). Among PCOS group, T2DM patients (594.6 \pm 156.12$)$ had significantly higher serum levels of ICAM-1 compared to IGT $(509.4 \pm 82.16 \mathrm{ng} / \mathrm{ml})$ and NGT $(281.3 \pm$ $95.29 \mathrm{ng} / \mathrm{ml}$ ) (Fig. 2b). In addition, IGT group had significantly higher expression and serum levels of ICAM-1 compared to NGT group (Fig. 1B and 2B).

\section{Correlation between serum and expression levels of} ICAM-1 with clinical and biochemical parameters of PCOS patients

In PCOS group, $(n=180)$, serum ICAM-1 levels were significantly positive correlated with of body composition parameters; BMI, waist/hip ratio, FMI\% and FFMI. Furthermore, serum ICAM-1 levels were significantly 
Table 2 Clinical, anthropometric and laboratory characteristics of PCOS groups

\begin{tabular}{|c|c|c|c|}
\hline \multirow[t]{2}{*}{ Parameters } & \multicolumn{3}{|l|}{ PCO patients $(n=180)$} \\
\hline & $\begin{array}{l}\text { NGT Group (mean } \pm \text { SD) } \\
(n=75)\end{array}$ & $\begin{array}{l}\text { IGT Group }(\text { mean } \pm \text { SD }) \\
(n=65)\end{array}$ & $\begin{array}{l}\text { T2DM Group (mean } \pm \text { SD) } \\
(n=40)\end{array}$ \\
\hline Systolic blood pressure (mm Hg) & $127.76 \pm 7.21$ & $134.49 \pm 4.74^{\mathrm{a}}$ & $139.67 \pm 7.04^{\mathrm{b}, \mathrm{c}}$ \\
\hline Diastolic blood pressure (mm Hg) & $83.8 \pm 3.86$ & $87.85 \pm 6.33^{\mathrm{a}}$ & $90.98 \pm 5.80^{b, c}$ \\
\hline Hirsutism score & $13.78 \pm 2.594$ & $13.42 \pm 2.9$ & $13.29 \pm 1.42$ \\
\hline Body mass index (kg/m2) & $32.96 \pm 14.25$ & $30.11 \pm 4.41$ & $32.65 \pm 5.73$ \\
\hline Waist/hip ratio & $0.96 \pm 0.212$ & $1.34 \pm 0.27^{\mathrm{a}}$ & $1.17 \pm 0.27^{b, c}$ \\
\hline Ovarian volume & $9.88 \pm 0.491$ & $10.75 \pm 1.21^{\mathrm{a}}$ & $11.89 \pm 1.4^{\mathrm{b}, \mathrm{c}}$ \\
\hline AFC & $11.38 \pm 0.491$ & $12.25 \pm 1.17^{\mathrm{a}}$ & $13.39 \pm 1.6^{b, c}$ \\
\hline FMI (kg/m2) & $6.20 \pm 0.68$ & $8.7 \pm 0.63^{\mathrm{a}}$ & $9.49 \pm 0.63^{b, c}$ \\
\hline FFMI (kg/m2) & $17.65 \pm 1.96$ & $25.02 \pm 1.82^{\mathrm{a}}$ & $27.17 \pm 1.06^{\mathrm{b}, \mathrm{c}}$ \\
\hline Total cholesterol (mg/dL) & $191.21 \pm 54.43$ & $201.5 \pm 54.43$ & $209.02 \pm 43.32$ \\
\hline Triglycerides (mg/dL) & $174.02 \pm 55.3$ & $184.2 \pm 57.19$ & $216.04 \pm 73.1^{b, c}$ \\
\hline LDL cholesterol (mg/dL) & $168.15 \pm 61.11$ & $163.17 \pm 57.96$ & $166.46 \pm 63.11$ \\
\hline HDL cholesterol (mg/dL) & $41.46 \pm 11.78$ & $36.21 \pm 13.85^{\mathrm{a}}$ & $39.8 \pm 15.8$ \\
\hline FPG (mg/dL) & $91.1 \pm 6.84$ & $108.16 \pm 12.50^{\mathrm{a}}$ & $140.5 \pm 12.7^{\mathrm{b}, \mathrm{c}}$ \\
\hline 2-h blood glucose (mg/dL) & $130.28 \pm 23.2$ & $171.94 \pm 28.68^{a}$ & $212.8 \pm 30.1^{b, c}$ \\
\hline FSI (IU/mL) & $13.5 \pm 3.289$ & $26.96 \pm 5.51^{a}$ & $21.1 \pm 3.34^{b, c}$ \\
\hline HOMA-IR & $2.85 \pm .855$ & $6.25 \pm 1.44^{\mathrm{a}}$ & $5.34 \pm 0.647^{\mathrm{b}, \mathrm{c}}$ \\
\hline HOMA- $\beta$ & $139.23 \pm 54.11$ & $124.4 \pm 35.3^{\mathrm{a}}$ & $108.6 \pm 41.55^{\mathrm{b}, \mathrm{c}}$ \\
\hline $\mathrm{HbA1c}(\%)$ & $5.66 \pm 0.35$ & $6.37 \pm 0.615^{\mathrm{a}}$ & $7.62 \pm 0.983^{b, c}$ \\
\hline Total testosterone $(\mathrm{ng} / \mathrm{mL})$ & $0.9 \pm 0.231$ & $0.99 \pm 0.304^{a}$ & $1.07 \pm 0.155^{b, c}$ \\
\hline Free androgen index & $1.813 \pm 0.98$ & $2.88 \pm 0.81^{\mathrm{a}}$ & $3.4 \pm 0.69^{b, c}$ \\
\hline $\mathrm{FSH}(\mathrm{mlU} / \mathrm{mL})$ & $6.02 \pm 1.314$ & $7.107 \pm 1.06^{\mathrm{a}}$ & $7.05 \pm 0.985^{b}$ \\
\hline $\mathrm{LH}(\mathrm{mlU} / \mathrm{mL})$ & $9.45 \pm 0.491$ & $10.44 \pm 1.15^{\mathrm{a}}$ & $11.98 \pm 0.201^{\mathrm{b}, \mathrm{c}}$ \\
\hline $\mathrm{LH} / \mathrm{FSH}$ & $1.63 \pm 0.341$ & $1.48 \pm 0.144^{\mathrm{a}}$ & $1.73 \pm 0.241^{c}$ \\
\hline SHBG (nmol/L) & $33.54 \pm 3.108$ & $26.23 \pm 2.011^{\mathrm{a}}$ & $24.197 \pm 0.96^{b, c}$ \\
\hline DHEA-S (mg/mL) & $2.17 \pm 0.245$ & $2.43 \pm 0.241^{a}$ & $2.66 \pm 0.286^{\mathrm{b}, \mathrm{c}}$ \\
\hline Androstenedione (ng/mL) & $1.23 \pm 0.371$ & $1.91 \pm 0.539^{a}$ & $1.88 \pm 0.43$ \\
\hline hs-CRP ( $\mu \mathrm{g} / \mathrm{ml})$ & $4.92 \pm 0.761$ & $5.79 \pm 0.760^{\mathrm{a}}$ & $6.37 \pm 0.91^{b, c}$ \\
\hline WBC count $\left(\right.$ cell $\left.\times 10^{3} / \mu \mathrm{l}\right)$ & $4.79 \pm 0.713$ & $8.45 \pm 2.27^{\mathrm{a}}$ & $8.58 \pm 1.281^{b, c}$ \\
\hline CIMT (mm) & $0.96 \pm 0.263$ & $1.09 \pm 0.36^{\mathrm{a}}$ & $1.74 \pm 0.467^{b, c}$ \\
\hline Serum ICAM-1 (ng/ml) & $281.3 \pm 95.29$ & $509.4 \pm 82.16^{a}$ & $594.6 \pm 156.12^{b, c}$ \\
\hline Relative ICAM expression levels & $2.68 \pm 0.85$ & $5.7 \pm 1.52^{\mathrm{a}}$ & $6.05 \pm 2.119^{b, c}$ \\
\hline
\end{tabular}

NGT Normal glucose tolerance, IGT impaired glucose tolerance, T2DM type 2 diabetes mellitus, FSI fasting serum insulin, FPG fasting plasma glucose, AFC antral follicle cells, FMI fat mass index, FFMI fat free mass index, HOMA-IR homeostasis model assessments of insulin resistance, DHEA dehydroepiandrosterone a significant difference between IGT vs NGT

${ }^{\mathrm{b}}$ significant difference between T2DM vs NGT., ${ }^{c}$ significant difference between T2DM vs IGT,

positive correlated with PCOS markers; ovarian volume, AFC, total testosterone, FAI, FSH, LH, LH/FSH, DHEA$\mathrm{S}$, and androstenedione. Even more interestingly, serum ICAM-1 levels were significantly positive correlated with cardio metabolic factors; FPG, 2-h blood glucose, FSI, HOMA-IR, HbA1c, hs-CRP, WBC count and CIMT. On the contrary, serum ICAM-1 levels were negatively correlated with HOMA- $\beta$ and SHBG (Table 3).
Regarding ICAM-1expression levels, there were significantly positive correlation with BMI, waist/hip ratio, FMI\% and FFMI, AFC, FPG, 2-h blood glucose, FSI, HOMA-IR, HbA1c, total testosterone, FAI, FSH, LH, LH/FSH, DHEA-S, and androstenedione, hs-CRP, WBC count and CIMT. On the contrary, the expression levels of ICAM- 1 were negatively correlated with HOMA- $\beta$ and SHBG (Table 3). 


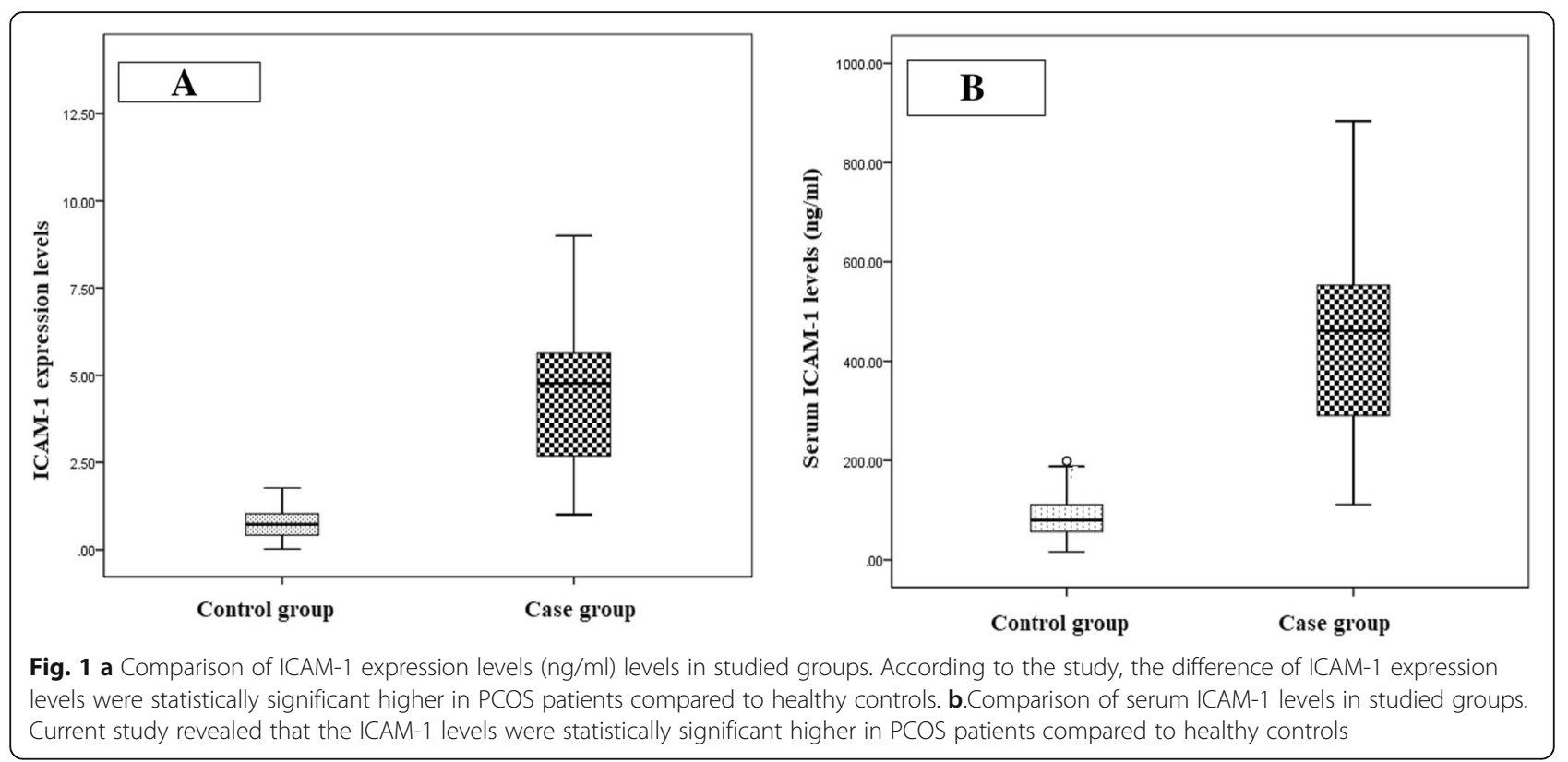

\section{Linear regression analysis with serum ICAM-1 levels as} dependent variable in PCOS groups

In PCOS group, linear regression analysis revealed that only HOMA-IR was the main predictor of serum ICAM-1 levels among other clinical and laboratory biomarkers of PCOS (Table 4).

\section{Linear regression analysis with ICAM-1 expression levels} as dependent variable in PCOS groups

In PCOS group, linear regression analysis showed that HOMA-IR, WBC count, and ovarian volume were the main predictors of ICAM-1 expression levels among other clinical and laboratory biomarkers of PCOS (Table 5).

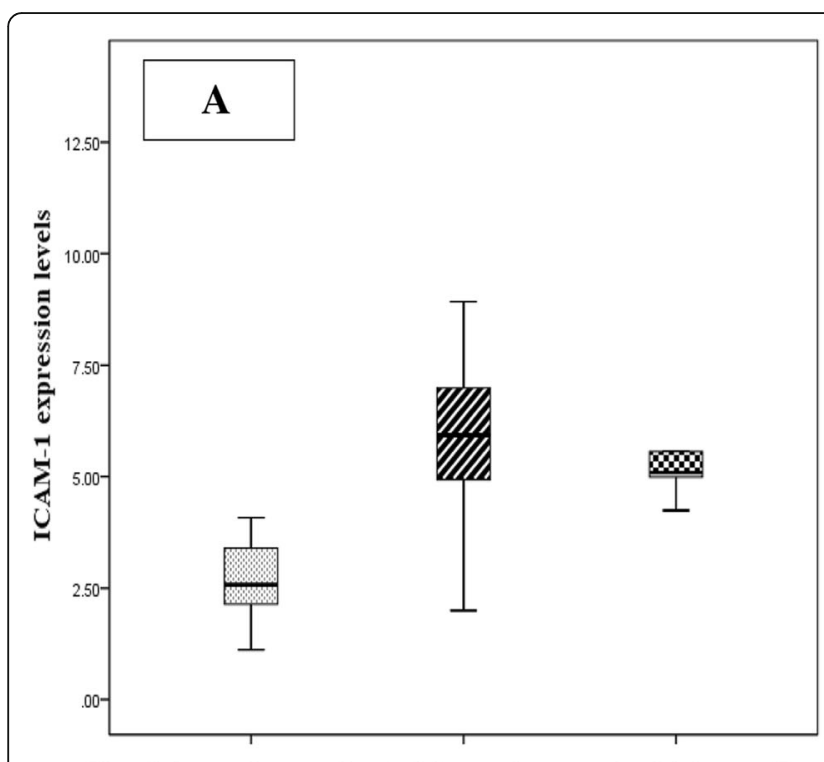

Normal glucose tolerance Impaired glucose tolerance Type 2 diabetes mellitus

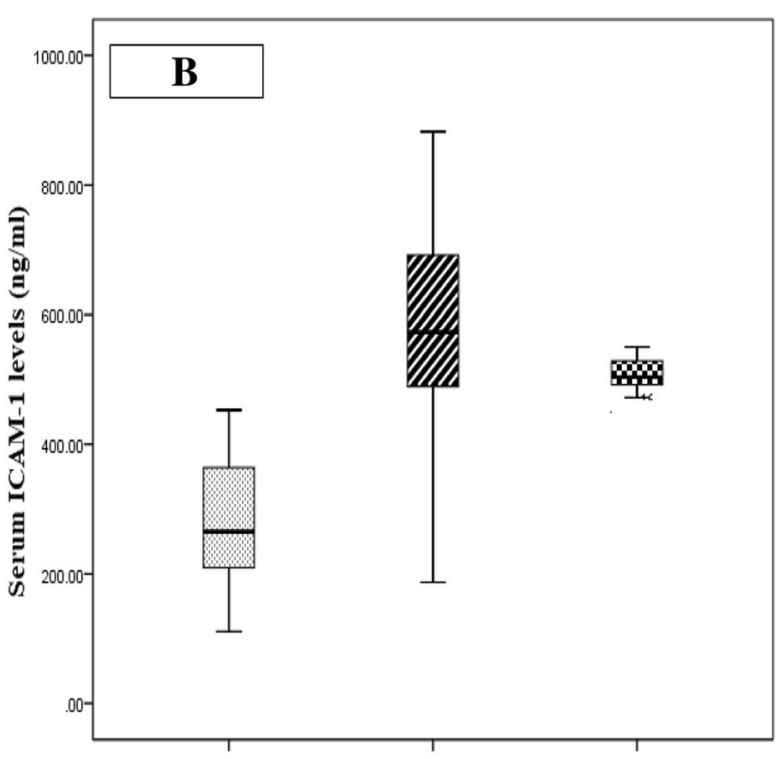

Normal glucose tolerance Impaired glucose tolerance Type 2 diabetes mellitus

Fig. 2 a Comparison of ICAM-1 expression levels in PCOS groups. Our study found that the ICAM-1 expression levels were statistically significant higher in PCOS patients with IGT compared to other PCOS groups. b Comparison of serum ICAM-1 levels (ng/ml) levels in PCOS groups. Our study found that the ICAM-1 serum levels were statistically significant higher in PCOS patients with IGT compared to other PCOS groups 
Table 3 Pearson correlation of serum ICAM-1 ( $\mathrm{ng} / \mathrm{ml}$ ) and ICAM-1 expression levels with clinical, anthropometric as well as biochemical characteristics in PCOS group

\begin{tabular}{|c|c|c|c|c|}
\hline \multirow[t]{2}{*}{ Characteristics } & \multicolumn{2}{|c|}{ Serum ICAM-1 levels } & \multicolumn{2}{|c|}{ ICAM-1 expression levels } \\
\hline & $r$ & $p$ & $r$ & $\mathrm{p}$ \\
\hline Hirsutism score & 0.095 & 0.205 & $0.095-$ & 0.210 \\
\hline Body mass index (kg/m2) & 0.086 & 0.253 & 0.073 & 0.332 \\
\hline Waist/hip ratio & 0.494 & $<0.001^{*}$ & 0.501 & $<0.001^{*}$ \\
\hline Ovarian volume & 0.187 & $0.012^{*}$ & 0.180 & 0.016 \\
\hline AFC & 0.187 & $<0.001^{*}$ & 0.180 & $<0.001^{*}$ \\
\hline FMl\% & 0.689 & $<0.001^{*}$ & 0.709 & $<0.001^{*}$ \\
\hline FFMI\% & 0.677 & $<0.001^{*}$ & 0.666 & $<0.001^{*}$ \\
\hline Total cholesterol (mg/dL) & 0.111 & 0.137 & 0.114 & 0.128 \\
\hline Triglycerides (mg/dL) & 0.019 & 0.797 & 0.030 & 0.694 \\
\hline LDL cholesterol (mg/dL) & 0.014 & 0.853 & 0.008 & 0.912 \\
\hline HDL cholesterol (mg/dL) & 0.036 & 0.631 & 0.044 & 0.561 \\
\hline $\mathrm{FPG}(\mathrm{mg} / \mathrm{dL})$ & 0.435 & $<0.001^{*}$ & 0.477 & $<0.001^{*}$ \\
\hline 2-h blood glucose (mg/dL) & 0.471 & $<0.001^{*}$ & 0.438 & $<0.001^{*}$ \\
\hline $\mathrm{FSI}(\mathrm{IU} / \mathrm{mL})$ & 0.854 & $<0.001^{*}$ & 0.794 & $<0.001^{*}$ \\
\hline HOMA-IR & 0.874 & $<0.001^{*}$ & 0.932 & $<0.001^{*}$ \\
\hline HbA1c (\%) & 0.404 & $<0.001^{*}$ & 0.369 & $<0.001^{*}$ \\
\hline HOMA- $\beta$ & -0.147 & $<0.001^{*}$ & 0.222 & $<0.001^{*}$ \\
\hline Total testosterone $(\mathrm{ng} / \mathrm{mL})$ & 0.114 & $<0.001^{*}$ & 0.121 & $<0.001^{*}$ \\
\hline Free androgen index & 0.466 & $<0.001^{*}$ & 0.469 & $<0.001^{*}$ \\
\hline FSH (mIU/mL) & 0.502 & $<0.001^{*}$ & 0.518 & $<0.001^{*}$ \\
\hline $\mathrm{LH}(\mathrm{mlU} / \mathrm{mL})$ & 0.549 & $<0.001^{*}$ & 0.561 & $<0.001^{*}$ \\
\hline SHBG (nmol/L) & -0.658 & $<0.001^{*}$ & 0.639 & $<0.001^{*}$ \\
\hline DHEA-S (mg/mL) & 0.269 & $<0.001^{*}$ & 0.262 & $<0.001^{*}$ \\
\hline Androstenedione (ng/mL) & 0.613 & $<0.001^{*}$ & 0.625 & $<0.001^{*}$ \\
\hline hs-CRP $(\mu \mathrm{g} / \mathrm{ml})$ & 0.342 & $<0.001^{*}$ & 0.374 & $<0.001^{*}$ \\
\hline 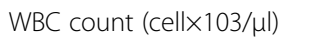 & 0.602 & $<0.001^{*}$ & 0.650 & $<0.001^{*}$ \\
\hline CIMT (mm) & 0.252 & $<0.001^{*}$ & 0.257 & $<0.001^{*}$ \\
\hline
\end{tabular}

FSI fasting serum insulin, FPG fasting plasma glucose, AFC antral follicle cells, FMI fat mass index, FFMI fat free mass index, HOMA-IR homeostasis model assessments of insulin resistance, $D H E A-S$ dehydroepiandrosteron sulfate e;. ${ }^{*} P<0.05$ when compared with control group

\section{Accuracy of serum and expression levels of ICAM-1 for diagnosis of PCOS by ROC analysis}

The power of serum ICAM-1 (ng/ml) levels to diagnose PCOS among studied subjects was evaluated using ROC analysis. The AUC was 0.988 (95\% CI =0.980-0.997) with sensitivity $=99.4 \%$, specificity $=85 \%$, and the cutoff values (121.2). When compared PCO patients to control, (Fig. 3a).

Regarding relative expression levels of ICAM-1, the AUC to diagnose PCOS among studied subjects was 0.991 (95\% CI $=0.983-0.999)$ with sensitivity $=99.4 \%$, specificity $=87.5 \%$, and the cutoff values (1.11), (Fig. 3b).

ROC analysis revealed that combined serum ICAM1 and expression levels to discriminate PCOS from control, the AUC was 0.989 (95\% CI 5 0.980-0.997, $P<0.001)$ with sensitivity $=99.4 \%$, specificity $=85.8 \%$, (Fig. 3c).
Accuracy of serum and expression levels of ICAM-1 for discriminating T2DM from NGT PCOS patients by ROC analysis

Among PCOS patients, the power of serum ICAM-1 (ng/ ml) levels in discriminating T2DM from NGT, the AUC was $0.956(95 \% \mathrm{CI}=0.933-0.986)$ with sensitivity $=93.3 \%$, specificity $=82.9 \%$, and the cutoff values (121.2). (Fig. 4a).

Regarding relative expression levels of ICAM-1, the AUC to diagnose PCOS among studied subjects was $0.976(95 \% \mathrm{CI}=0.951-1.000)$ with sensitivity $=95.2 \%$, specificity $=99.3 \%$, and the cutoff values (1.11), (Fig. 4b).

ROC analysis revealed that combined serum and expression levels of ICAM-1 to discriminate PCOS from control, the AUC was 0.963 (95\% CI $=0.937-$ $0.988, P<0.001)$ with sensitivity $=98.1 \%$, specificity $=$ $69.9 \%$ (Fig. 3c). 
Table 4 linear regression analyses in PCOS women to test the influence of the main independent variables against serum ICAM-1 levels (dependent variable) in PCOS women

\begin{tabular}{|c|c|c|c|c|c|c|c|c|}
\hline \multicolumn{2}{|c|}{ Model } & \multicolumn{2}{|c|}{ Unstandardized Coefficients } & \multirow{2}{*}{$\begin{array}{l}\text { Standardized Coefficients } \\
\text { Beta }\end{array}$} & \multirow[t]{2}{*}{$\mathrm{t}$} & \multirow[t]{2}{*}{$P$ value } & \multicolumn{2}{|l|}{ 95\% C.I. } \\
\hline & & B & SE & & & & Lower Bound & Upper Bound \\
\hline \multirow[t]{12}{*}{1} & (Constant) & 135.851 & 80.832 & & 1.681 & 0.095 & $-23.727-$ & 295.428 \\
\hline & Total cholesterol & $-0.019-$ & 0.103 & $0.006-$ & 0.190 & 0.850 & $-0.222-$ & 0.183 \\
\hline & LDL cholesterol & $-0.001-$ & 0.087 & 0.000 & 0.014 & 0.989 & $-0.172-$ & 0.170 \\
\hline & $\mathrm{FPG}$ & $-0.163-$ & 0.456 & 0.019 & 0.357 & 0.721 & $-1.063-$ & 0.737 \\
\hline & HOMA-IR & 90.239 & 4.100 & 0.917 & 22.008 & $<0.001^{*}$ & 82.144 & 98.334 \\
\hline & Body mass index & $-0.215-$ & 0.537 & $0.012-$ & 0.400 & 0.689 & $-1.274-$ & 0.844 \\
\hline & $\mathrm{LH}$ & 1.267 & 7.087 & 0.008 & 0.179 & 0.858 & $-12.725-$ & 15.259 \\
\hline & hs-CRP & $-4.453-$ & 7.328 & 0.024 & 0.608 & 0.544 & $-18.919-$ & 10.014 \\
\hline & WBC count & 2.683 & 3.020 & 0.034 & 0.888 & 0.376 & $-3.280-$ & 8.645 \\
\hline & Ovarian volume & $-7.053-$ & 5.234 & 0.050 & 1.347 & 0.180 & $-17.386-$ & 3.280 \\
\hline & Hirsutism score & $-2.981-$ & 2.232 & 0.040 & 1.336 & 0.184 & $-7.388-$ & 1.426 \\
\hline & CIMT & 23.455 & 15.458 & 0.059 & 1.517 & 0.131 & $-7.061-$ & 53.972 \\
\hline
\end{tabular}

\section{Discussion}

Because current treatment of PCOS is still suboptimal, and cardio-metabolic diseases is the common cause of death all over the world among PCO cases, early assessment of levels of cytokines could potentially help in early diagnosis and prevention of these common diseases To the best of our knowledge, this is the first study regarding for the association between ICAM-1serum level and gene expression as cardiovascular predictors with PCOS as well as its clinic-morphological features.

In order to assess the risk of cardio-vascular disease among PCOS, our results revealed that the cardiometabolic factors; systolic blood pressure, TC, TG, LDL cholesterol, 2-h plasma glucose, FSI, HbA1c, HOMAIR,,FPG and CIMT were significantly higher in PCOS compared to control group. Our previous experience also suggested that PCOS women had higher values of parameters of hyperglycemia and dyslipidemia as well as fat mass $[18,19]$.

Similar to our results, Glueck et al. found that PCOS patients are at an increased risk of metabolic syndrome and cardiovascular risk factors in later life [20]. Similar results confirmed by, Zhao et al. they observed high risk of coronary heart disease in PCOS [21]. Moreover, Morgan et al. study detected higher mortality in PCOS compared to control group [22].. On the contrary, Pierpoint et al. [23]

Table 5 linear regression analyses in PCOS women to test the influence of the main independent variables against ICAM-1 gene expression (dependent variable) in PCOS women

\begin{tabular}{|c|c|c|c|c|c|c|c|c|}
\hline \multirow{2}{*}{\multicolumn{2}{|c|}{ Model }} & \multicolumn{2}{|c|}{ Unstandardized Coefficients } & \multirow{2}{*}{$\begin{array}{l}\text { Standardized Coefficients } \\
\text { Beta }\end{array}$} & \multirow[t]{2}{*}{$t$} & \multirow[t]{2}{*}{$P$ value } & \multicolumn{2}{|l|}{ 95\% C.I. } \\
\hline & & B & SE & & & & Lower Bound & Upper Bound \\
\hline \multirow[t]{12}{*}{1} & (Constant) & -0.928 & 1.221 & & 0.760 & 0.448 & -3.340 & 1.483 \\
\hline & Total cholesterol & -0.001 & 0.002 & 0.017 & 0.456 & 0.649 & -0.004 & 0.002 \\
\hline & LDL cholesterol & 0.000 & 0.001 & 0.012 & 0.340 & 0.734 & -0.002 & 0.003 \\
\hline & FPG & 0.004 & 0.007 & 0.036 & 0.526 & 0.599 & -0.010 & 0.017 \\
\hline & HOMA-IR & 0.829 & 0.062 & 0.720 & 13.389 & $<0.001^{*}$ & 0.707 & 0.952 \\
\hline & Body mass index & 0.000 & 0.008 & 0.002 & 0.052 & 0.958 & -0.016 & 0.016 \\
\hline & $\mathrm{LH}$ & 0.114 & 0.107 & 0.065 & 1.069 & 0.287 & -0.097 & 0.326 \\
\hline & hs-CRP & 0.139 & 0.111 & 0.063 & 1.251 & 0.213 & -0.080 & 0.357 \\
\hline & WBC count & 0.152 & 0.046 & 0.162 & 3.328 & $0.001^{*}$ & 0.062 & 0.242 \\
\hline & Ovarian volume & $-0.178-$ & 0.079 & 0.0107 & 2.247 & $0.026^{*}$ & -0.334 & -0.022 \\
\hline & Hirsutism score & 0.012 & 0.034 & 0.014 & 0.368 & 0.713 & -0.054 & 0.079 \\
\hline & CIMT & 0.039 & 0.234 & 0.008 & 0.168 & 0.867 & -0.422 & 0.500 \\
\hline
\end{tabular}



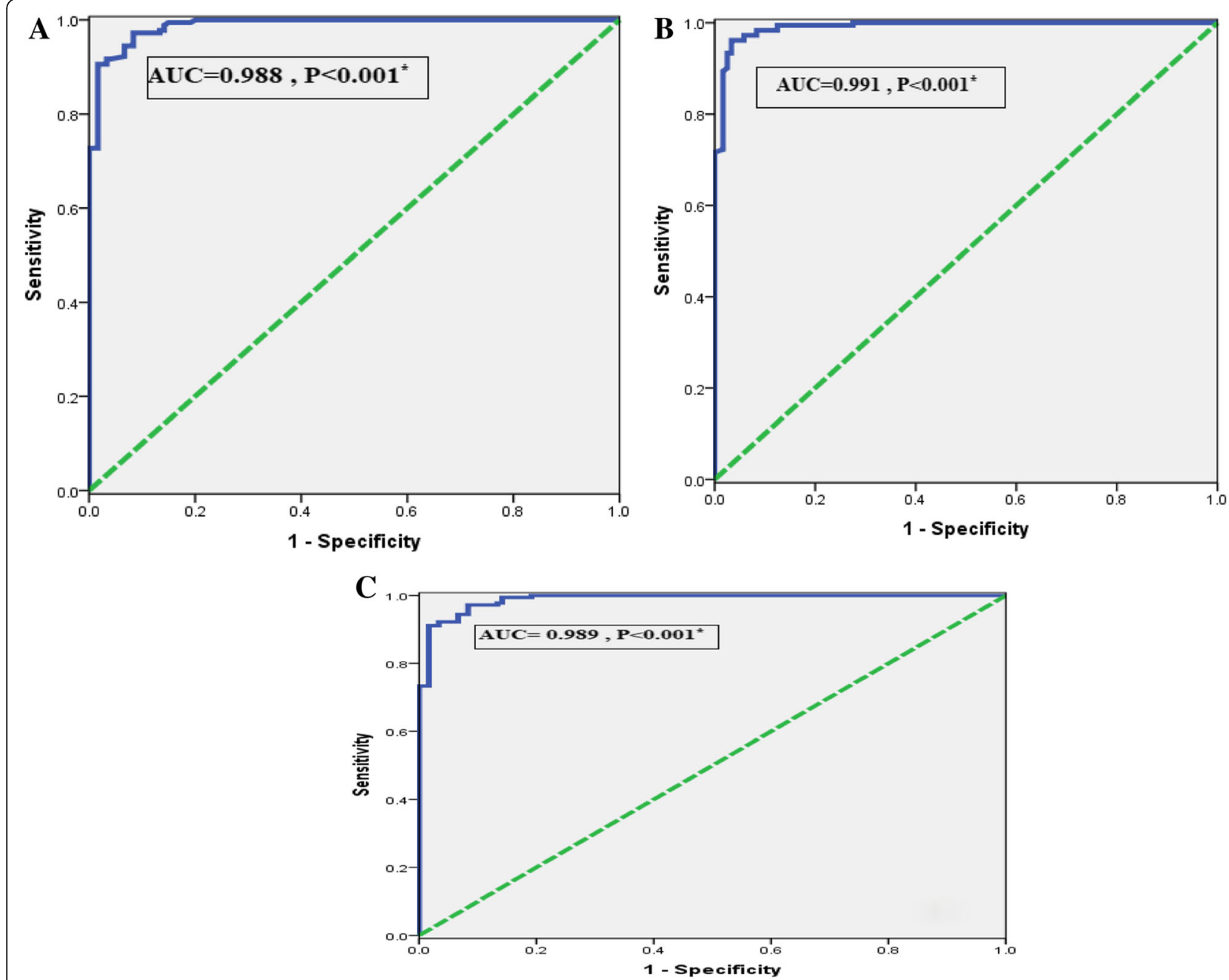

Fig. 3 a ROC curve of serum ICAM-1 for diagnosis PCOS. b ROC curve of ICAM-1 expression levels for diagnosis PCOS patients. $\mathbf{c}$ ROC curve for combination of ICAM-1 expression and serum levels for diagnosis PCOS patients

and Wild et al. [24] conducted their studies on CAD morbidity or mortality among PCOS patients, and they did not find any significant association between PCOS and CAD morbidity and/or mortality.

Regarding lipid profile in PCOS women, similar to our resultsWild et al. Showed that women with PCOS have higher levels of TG, LDL-cholesterol and TC, and lower HDL cholesterol levels compared with control group [25]. In addition, Mulhim et al. conducted their study to assess metabolic and clinical Profile of Saudi patients with PCOS, they found that PCOS patients had non- significantly difference in TC, HDL cholesterol, TG, LDL cholesterol, or FPG values compared to control group [26].

Regarding association of obesity and PCOS as cardiovascular risk, the study conducted by Stepto et al. demonsterated that the influence of obesity on PCOS clinical and biochemical characteristics was nonsignificant [27].
Our finding adds to the growing body of evidence implicating that both expression and serum levels of ICAM-1 had an important role in pathogenesis of atherosclerosis as well as PCOS. The current study revealed that ICAM-1 expression and serum levels were significant higher in PCOS patients as compared to control group. Moreover, among PCOS group, T2DM patients had significantly higher expression and serum levels of ICAM-1 in comparison to IGT and NGT. In agreement with our results, Diamanti-Kandrakis et al. proposed that levels of adhesion molecules were higher, including ICAM-1, in PCOS patients compared to healthy women [28].

Similar to our data, Danesh et al. and his colleagues observed that insulin resistance has a central role in chronic inflammation and endothelial dysfunction, which represent an early sign of atherosclerosis [29]. In addition, study conducted by Hak et al. observed higher levels of ICAM-1 in nondiabetic subjects with 

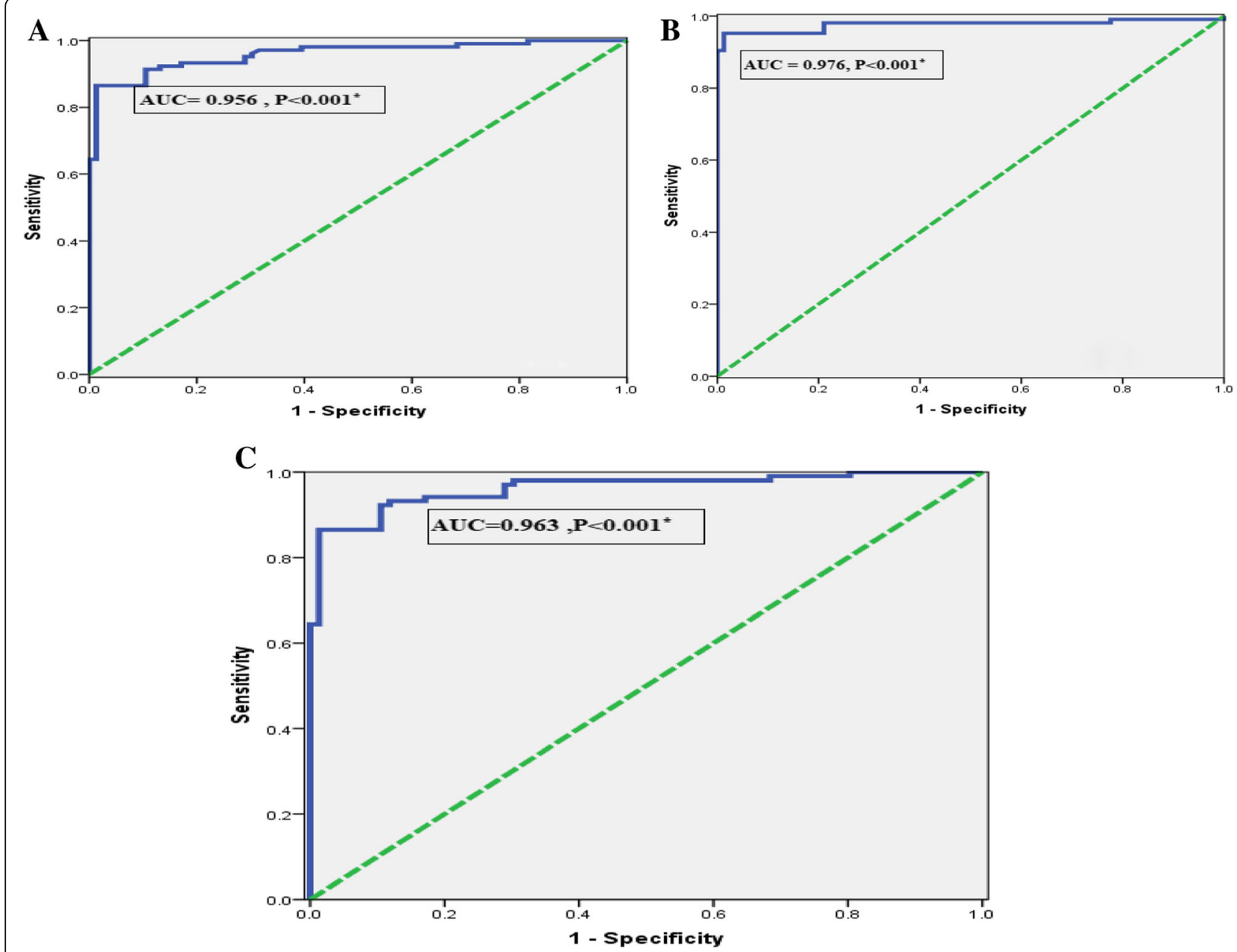

Fig. 4 a ROC curve of serum ICAM-1 for discriminating T2DM from NGT patients with PCOS. b ROC curve of serum ICAM-1 for discriminating T2DM from NGT patients with PCOS. c ROC curve for combination of ICAM-1 expression and serum levels for discriminating T2DM from NGT patients with PCOS

insulin resistance [30]. Also, regarding the association of T2DM and ICAM-1 expression level, Kado et al. detected higher levels of ICAM-1 expression in hyperglycemia [31]. Similar results found higher adhesion molecules levels in diabetic patients [32, 33],

Our study evaluated the correlations of ICAM-1expression and serum levels, we found that both expression and serum levels were significantly positive correlated with cardiovascular risk and PCOS phenotypes among PCOS patients. Moreover, in order to better elucidate the association of ICAM -1serum levels, linear regression test was done and we observed among clinical and laboratory markers of PCOS, only HOMA-IR was the main predictors of serum ICAM-1 levels in PCOS patients. While, HOMA-IR, WBC count, and ovarian volume were the main predictors of ICAM-1 expression levels among PCOS cases. Similarly, Blankenberg et al. showed positive correlation between cellular adhesion molecules and markers of low-grade inflammation and endothelial dysfunction [34].
A noted feature of PCOS pathogenic process is the role of inflammation. Here presented data revealed higher levels of WBC count and hs-CRP in PCOS patients. Similarly, in earlier published studies, we found that PCOS women had higher levels of inflammatory markers $[35,36]$.

One of the main objectives of the current study was to evaluate association between PCOS clinical and laboratory markers with atherosclerosis. We studied CIMT as a marker of pre-clinical atherosclerosis. We found that CIMT was significant high in PCOS patients compared to control group. Moreover, among PCOS group, T2DM patients had significantly higher expression and serum values of CIMT compared to IGT and NGT. In addition, our study revealed significantly positive correlation between CIMT with cardiovascular risk and PCOS phenotypes in PCOS patients. In agreement to our results, Rubio-Guerra et al. CIMT values were positively correlated with circulating adhesion molecules [37]. Similarly, 
Tardif et al. study suggested that, increased CIMT is independently linked to cardiovascular risks [38]. Notably, also systematic review and meta-analysis confirmed that CIMT could be used diagnostic marker of risk for cardiovascular events [39, 40, 41]. In contrast, Talbott et al. found that after adjusting for age and BMI, PCOS was not a significant predictor of CIMT [42].

In attempt to assess the diagnostic power of ICAM-1 expression and serum levels we evaluated our results using ROC analysis. We observed that the AUC of expression levels of ICAM-1 was higher than serum levels of ICAM-1 in diagnosis of PCOS as well as in discriminating T2DM from NGT patients with PCOS. Interestingly, when we combined diagnostic power of expression and serum levels of ICAM-1 the AUC of ICAM-1 serum levels increased.

\section{Conclusion}

We found that expression and serum levels of ICAM1were higher in PCOS patients mainly T2DM. Moreover, they were significantly positive correlated with cardiovascular risk and PCOS phenotypes. Interestingly, combination of both ICAM-1 expression and serum levels improved the diagnostic values of serum ICAM-1. thus, the expression and serum levels of ICAM-1could be a useful diagnostic biomarker of PCOS .

\section{Acknowledgements}

Not applicable.

\section{Authors' contributions}

NMR, HGA, WME, KFH,AMKA, HMA, MER,MSB, AEA, MSY and, MAF collected patients' samples and clinical data. Amal S. El-Shal prepared sample for laboratory investigations. Nearmeen M. Rashad wrote the paper. Statistical analysis, interpretation of data and preparation the paper for submitting international was done by Nearmeen M. Rashad. Critical revision of the manuscript was performed by all of the authors. All authors read and approved the final manuscript.

\section{Funding}

No fund.

\section{Availability of data and materials}

Data available on demand.

\section{Consent for publication}

Not applicable.

\section{Competing interests}

The authors declare that they have no competing interests.

\footnotetext{
Author details

'Internal Medicine Department, Faculty of Medicine, Zagazig University, Zagazig, Egypt. ${ }^{2}$ Medical Biochemistry Department, Faculty of Medicine, Zagazig University, Zagazig, Egypt. ${ }^{3}$ Cardiology Department, Faculty of Medicine, Zagazig University, Zagazig, Egypt. ${ }^{4}$ Obstetrics and Gynecology Department, Faculty of Medicine, Zagazig University, Zagazig, Egypt. ${ }^{5}$ Physiology Department, Faculty of Medicine-Zagazig University, Zagazig, Egypt.
}

Received: 28 May 2019 Accepted: 10 September 2019

Published online: 19 October 2019

\section{References}

1. Kauffman RP, Baker TE, Baker VM, DiMarino P, Castracane D. Endocrine and metabolic differences among phenotypic expressions of polycystic ovary syndrome according to the 2003 Rotterdam consensus criteria. Am J Obstet Gynecol. 2008;198(6):670.e1-670.e10.

2. Malik S, Wong ND. Metabolic syndrome, cardiovascular risk and screening for subclinical atherosclerosis. Expert Rev Cardiovasc Ther. 2009;7(3):273-80. https://doi.org/10.1586/14779072.7.3.273.

3. Celermajer DS, Ayer JG. Childhood risk factors for adult cardiovascular disease and primary prevention in childhood. Heart. 2006;92:1701-6.

4. Oskui PM, French WJ, Herring MJ, Mayeda GS, Burstein S, Kloner RA. Testosterone and the cardiovascular system: a comprehensive review of the clinical literature. J Am Heart Assoc. 2013;2:e000272.

5. Lim SS, Davies MJ, Norman RJ, Moran LJ. Overweight, obesity and central obesity in women with polycystic ovary syndrome: a systematic review and meta-analysis. Hum Reprod Update. 2012;18:618-37.

6. Tanaka H, Dinenno FA, Monahan KD, DeSouza CA, Seals DR. Carotid artery wall hypertrophy with age is related to local systolic blood pressure in healthy men. Arterioscler Thromb Vasc Biol. 2001;21:82-7.

7. Schmidt Trucksass A, Grathwohl D, Schmid A, Boragk R, Upmeier C, Keul J, Huonker M. Structural, functional, and hemodynamic changes of the common carotid artery with age in male subjects. Arterioscler Thromb Vasc Biol. 1999;19:1091-7.

8. Bianchini E, Bozec E, Gemignani V, Faita F, Giannarelli C, Ghiadoni L, Demi $M$, Boutouyrie P, Laurent S. Assessment of carotid stiffness and intima-media thickness from ultrasound data: comparison between two methods. J Ultrasound Med. 2010:29:1169-75.

9. Khodabandehlou K, Masehi-Lano JJ, Poon C, Wang J, Chung EJ. Targeting cell adhesion molecules with nanoparticles using in vivo and flow-based in vitro models of atherosclerosis. Exp Biol Med. 2017;242(8):799-812.

10. Libby P, Ross R. Atherosclerosis and coronary artery disease. Philadelphia: Lippincott-Raven; 1996. p. 585-94.

11. Vadas MA, Gamble JR. Endothelial adhesion molecules in atherogenesis. A concerto or a solo? Circ Res. 1996;79:1216-7.

12. Diamanti-Kandrakis E, Paterakis T, Alexandraki K, Piperi C, Aessopos A, Katsikis I, et al. Indices of low-grade chronic inflammation in polycystic ovary syndrome and the benefical effect of metformin. Hum Reprod. 2006; 21:1426-31. https://doi.org/10.1093/humrep/del003.

13. Lawson C, Wolf S. ICAM-1 signaling in endothelial cells. Pharmacol Rep. 2009:61:22-32.

14. American Diabetes Association. Diagnosis and classification of diabetes mellitus. Diabetes Care. 2016;39(Suppl. 1):S1-2. https://doi.org/10.2337/dc16-S001.

15. The Rotterdam ESHRE-ASRM-Sponsored PCOS Consensus Workshop Group. Revised 2003 consensus on diagnostic criteria and long-term health risks related to polycystic ovary syndrome (PCOS). Hum Reprod. 2004;19:41-7.

16. Friedewald WT, Levy RI, Fredrickson DS. Estimation of the concentration of low-density lipoprotein cholesterol in plasma, without use of the preparative ultracentrifuge. Clin Chem. 1972;18:499-502.

17. Sawa Y, Ueki T, Hata M, et al. LPS-induced IL-6, IL-8, VCAM-1, and ICAM-1 expression in human lymphatic endothelium. J Histochem Cytochem. 2008; 56(2):97-109. https://doi.org/10.1369/jhc.7A7299.2007.

18. El-Shal AS, Zidan HE, Rashad NM, Abdelaziz AM, Harira MM. Association between genes encoding components of the leutinizing hormone/ luteinizing hormone-choriogonadotrophin receptor pathway and polycystic ovary syndrome in Egyptian women. IUBMB Life. 2016;68(1):23-36.

19. El-Shal AS, Shalaby SM, Aly NM, Rashad NM, Abdelaziz AM. Genetic variation in the vitamin D receptor gene and vitamin D serum levels in Egyptian women with polycystic ovary syndrome. Mol Biol Rep. 2013;40(11):6063-73.

20. Glueck CJ, Woo JG, Khoury PR, Morrison JA, Daniels SR, Wang P. Adolescent oligomenorrhea (age 14-19) tracks into the third decade of life (age 20-28) and predicts increased cardiovascular risk factors and metabolic syndrome. Metabolism. 2015;64:539-53.

21. Zhao L, Zhu Z, Lou H, Zhu G, Huang W, Zhang S, Liu F. Polycystic ovary syndrome (PCOS) and the risk of coronary heart disease (CHD): a meta-analysis. Oncotarget. 2016;7:33715-21. https:/doi.org/10.18632/oncotarget.9553.

22. Morgan $\mathrm{CL}$, Jenkins-Jones $\mathrm{S}$, Currie $\mathrm{CJ}$, Rees DA. Evaluation of adverse outcome in young women with polycystic ovary syndrome versus matched 
reference controls: a retrospective, observational study. J Clin Endocrinol Metab. 2012;97:3251-60.

23. Pierpoint T, McKeigue PM, Isaacs AJ, Wild SH, Jacobs HS. Mortality of women with polycystic ovary syndrome at long-term follow-up. J Clin Epidemiol. 1998;51:581-6.

24. Wild S, Pierpoint T, McKeigue P, Jacobs H. Cardiovascular disease in women with polycystic ovary syndrome at long-term follow-up: a retrospective cohort study. Cli Endocrinol (Oxf). 2000;52:595-600.

25. Wild RA, Rizzo M, Clifton S, Carmina E. Lipid levels in polycystic ovary syndrome: systematic review and meta-analysis. Fertil Steril. 2011;95:1073-9.

26. Al Mulhim AA, Abul Heija AA, Al Talib AA, Al Turki HA, Gasim TG. Hormonal, metabolic and clinical profile of Saudi women with polycystic ovary syndrome. Saudi J Med Med Sci. 2013;1(1):30-4. https://doi.org/10.4103/1658-631X.112920.

27. Stepto NK, Cassar S, Joham AE, Hutchison SK, Harrison CL, Goldstein RF, et al. Women with polycystic ovary syndrome have intrinsic insulin resistance on euglycaemic-hyperinsulaemic clamp. Hum Reprod. 2013;28: 777-84. https://doi.org/10.1093/humrep/des463.

28. Kelly KA, Allport JR, Tsourkas A, Shinde-Patil VR, Josephson L, Weissleder R. Detection of vascular adhesion molecule-1 expression using a novel multimodal nanoparticle. Circ Res. 2005;96(3):327-36.

29. Danesh J, Whincup P, Walker M, Lennon L, Thomson A, Appleby P, et al. Low-grade inflammation and coronary heart disease: a prospective study and upregulated metaanalyses. Br Med J. 2000;321:199-204. https://doi.org/ 10.1136/bmj.321.7255.199.

30. Hak AE, Pols HA, Stehouwer CD, Meijer J, Kiliaan AJ, Hofman A, et al. Markers of inflammation and cellular adhesion molecules in relation to insulin resistance in nondiabetic elderly: the Rotterdam study. J Clin Endocrinol Metab. 2001;86:4398-405. https://doi.org/10.1210/jc.86.9.4398.

31. Kado S, Wakatsuki T, Yamamoto M, Nagato N. Expression of intercellular adhesion molecule-1 induced by high glucose in human aortic endothelial cells. Life Sci. 2001;68:727-37. https://doi.org/10.1016/S0024-3205(00)00968-1.

32. Sugimoto H, Shikata K, Hirata K, Akiyama K, Matsuda M, Kushiro M, et al. Increased expression of intercellular adhesion molecule-1 (ICAM-1) in diabetic rat glomeruli. Glomerular hyperfunction is a potential mechanism of ICAM-1 upregulation. Diabetes. 1997;46:2075-81. https:/doi.org/10.2337/diabetes.46.12.2075.

33. Fasching P, Veitl M, Rohac M, Streli C, Schneider B, WaldhausI W, et al. Elevated concentrations of circulating adhesion moolecules and their association with microvascular complications in insulin-dependent diabetes mellitus. J Clin Endocrinol Metab. 1996;81:4314-7. https://doi.org/10.1210/jc.81.12.4313.

34. Blankenberg S, Barbaux S, Tiret L. Adhesion molecules and atherosclerosis. Atherosclerosis. 2003;170:191-203. https:/doi.org/10.1016/50021-9150(03)00097-2.

35. Rashad NM, El-Shal AS, Amin Al, Soliman MH. Effects of probiotics supplementation on macrophage migration inhibitory factor and clinical laboratory feature of polycystic ovary syndrome. J Funct Foods. 2017;36:317-24.

36. Rashad NM, El-Shal AS, Abdelaziz AM. Association between inflammatory biomarker serum procalcitonin and obesity in women with polycystic ovary syndrome. J Reprod Immunol. 2013;97(2):232.

37. Rubio-Guerra AF, Vargas-Robles H, Serrano AM, Lozano-Nuevo JJ, Escalante-Acosta BA. Correlation between the levels of circulating adhesion molecules and atherosclerosis in type-2 diabetic normotensive patients. Cell Adhes Migr. 2009:3: 369-72. https//doi.org/10.4161/cam.3.4.9463.

38. Tardif JC, Heinonen T, Orloff D, Libby P. Vascular biomarkers and surrogates in cardiovascular disease. Circulation. 2006;113:2936-42.

39. Lorenz MW, Markus HS, Bots ML, Rosvall M, Sitzer M. Prediction of clinical cardiovascular events with carotid intima-media thickness: a systematic review and meta-analysis. Circulation. 2007;115:459-67.

40. Lorenz MW, Polak JF, Kavousi M, Mathiesen EB, Volzke H, et al. Carotid intima-media thickness progression to predict cardiovascular events in the general population (the PROG-IMT collaborative project): a meta-analysis of individual participant data. Lancet. 2012;379:2053-62.

41. de Groot E, van Leuven SI, Duivenvoorden R, Meuwese MC, Akdim F, et al. Measurement of carotid intima-media thickness to assess progression and regression of atherosclerosis. Nat Clin Pract Cardiovasc Med. 2008:5:280-8.

42. Talbott EO, Guzick DS, Sutton-Tyrrell K, McHugh-Pemu KP, Zborowski JV, Remsberg KE, et al. Evidence for association between polycystic ovary syndrome and premature carotid atherosclerosis in middle-aged women. Arterioscler Thromb Vasc Biol. 2000;20(11):2414-21.

\section{Publisher's Note}

Springer Nature remains neutral with regard to jurisdictional claims in published maps and institutional affiliations.

Ready to submit your research? Choose BMC and benefit from:

- fast, convenient online submission

- thorough peer review by experienced researchers in your field

- rapid publication on acceptance

- support for research data, including large and complex data types

- gold Open Access which fosters wider collaboration and increased citations

- maximum visibility for your research: over $100 \mathrm{M}$ website views per year

At BMC, research is always in progress.

Learn more biomedcentral.com/submissions 